\title{
Optimising Antimicrobial Selection and Duration in the Treatment of Febrile Neutropenia in Children
}

\author{
Jessica E Morgan (1D ${ }^{1,2}$ \\ Bob Phillips (1D) ${ }^{1,2}$ \\ Gabrielle M Haeusler (D) ${ }^{3-6}$ \\ Julia C Chisholm (iD)
}

'Centre for Reviews and Dissemination, University of York, Heslington, YOIO 5DD, UK; ${ }^{2}$ Department of Paediatric Oncology, Leeds Teaching Hospitals NHS Trust, Leeds, LSI 3EX, UK; ${ }^{3} \mathrm{NHMRC}$ National Centre for Infections in Cancer, Sir Peter MacCallum Department of Oncology, University of Melbourne, Parkville, Victoria, 3010, Australia; ${ }^{4}$ Sir Peter MacCallum Department of Oncology, University of Melbourne, Parkville, Victoria, 3010, Australia; ${ }^{5}$ Infection Diseases Unit, Department of General Medicine, Royal Children's Hospital, Parkville, Victoria, 3168, Australia; 'Murdoch Children's Research Institute, Parkville, Victoria, 3052, Australia; ${ }^{7}$ Royal Marsden Hospital and Institute of Cancer Research, Sutton, SM2 5PT, UK
Correspondence: Jessica E Morgan Centre for Reviews and Dissemination, University of York, Heslington, YOIO 5DD, UK

Tel +4401904321082

Email jess.morgan@york.ac.uk
This article was published in the following Dove Press journal: Infection and Drug Resistance

\begin{abstract}
Febrile neutropenia (FN) is a frequent complication of cancer treatment in children. Owing to the potential for overwhelming bacterial sepsis, the recognition and management of FN requires rapid implementation of evidenced-based management protocols. Treatment paradigms have progressed from hospitalisation with broad spectrum antibiotics for all patients, through to risk adapted approaches to management. Such risk adapted approaches aim to provide safe care through incorporating antimicrobial stewardship (AMS) principles such as implementation of comprehensive clinical pathways incorporating deescalation strategies with the imperative to reduce hospital stay and antibiotic exposure where possible in order to improve patient experience, reduce costs and diminish the risk of nosocomial infection. This review summarises the principles of risk stratification in FN, the current key considerations for optimising empiric antimicrobial selection including knowledge of antimicrobial resistance patterns and emerging technologies for rapid diagnosis of specific infections and summarises existing evidence on time to treatment, investigations required and duration of treatment. To aid treating physicians we suggest the key features based on current evidence that should be part of any FN management guideline and highlight areas for future research. The focus is on treatment of bacterial infections although fungal and viral infections are also important in this patient group.
\end{abstract}

Keywords: paediatric, febrile neutropenia, antimicrobials

\section{Plain Language Summary}

Children undergoing treatment for cancer are at risk of serious infections which may be seen as a fever with a low white blood cell count (neutropenia). This condition is called febrile neutropenia (FN). The treatment of $\mathrm{FN}$ has changed over time. In the past all patients were treated in hospital with antibiotics that cover a range of infections. Now, treatment depends on the chance that an individual child has a serious infection. This means for some children we can reduce the time spent in hospital and use fewer antibiotics. This article describes the research behind current best practice in the treatment of $\mathrm{FN}$ in children. We focus on:

- Recognising FN quickly.

- Starting treatment rapidly.

- Which antibiotics to use.

- How long to give them for.

We suggest the things that all health care workers should think about when treating children with FN and what further research is needed to improve care in the future. 


\section{Introduction}

Fever with neutropenia (febrile neutropenia; $\mathrm{FN}$ ) is one of the commonest complications in the treatment of childhood cancer and is a significant cause of hospitalisation with attendant disruption to the child and family, risks of nosocomial infection and associated healthcare costs. Bacteraemia is identified in $11-24 \%$ of children with FN and intensive care admission is reported in up to $11 \%$ of episodes with mortality rates of up to $3 \% .{ }^{1}$ Overall gram positive organisms tend to be identified in blood cultures slightly more commonly than gram negative organisms, however this ratio is influenced by the timing of blood cultures (i.e pre or post antibiotic), presence of central lines and concurrent antibacterial prophylaxis. ${ }^{2,3}$ In a large series of FN episodes in children with cancer, $59 \%$ of children had no documented clinical or microbiological evidence of infection, $24 \%$ had bacteraemia, $2 \%$ had a microbiologically documented infection without bacteraemia, $6 \%$ had a clinically documented infection and $9 \%$ had a fungal infection. ${ }^{4}$

Owing to the risk of bacteraemia, $\mathrm{FN}$ is traditionally managed by urgent hospital attendance and empirical broad spectrum intravenous antibiotics until resolution of fever with average inpatient stay reported as 5 days across many UK and Australian paediatric oncology settings. ${ }^{5-8}$ However, it has become apparent in recent years that FN episodes are heterogenous; the risk of significant infection varies among different patients and episodes and the approach of hospitalising all patients until resolution of fever and recovery of neutrophil count overtreats a significant group of lower risk patients, increasing hospital stay and costs and risking evolution of antimicrobial resistance. Current international guidance now recommends a risk-stratified approach to treatment of paediatric FN with the aim of improving patient experience and practising responsible antimicrobial stewardship (AMS) by limiting unnecessary or prolonged antibiotics in carefully selected patients. ${ }^{9}$

This article is focused on improving detection and optimising antibacterial management of FN and highlights recent literature in this area. An updated paediatric specific FN guideline is available elsewhere. ${ }^{9,10}$ Fungal and viral infections and their treatment will not be considered in detail and non-neutropenic fever is reviewed elsewhere. ${ }^{11,12}$ We will discuss optimising antimicrobial selection, reducing hospital stay, the role of biomarkers to predict infection risk and the role of new rapid diagnostic techniques. Many of the recommendations we discuss are informed by data from studies in high-income countries. Clinicians working in low and middle-income countries may need to consider other factors such as the prevalence of other specific infections (including malaria and other parasites), the availability of diagnostic testing, patient access to healthcare (including transport options), nutritional status of the population, the intensity of chemotherapy regimes delivered (with their associated infection risks), antibiotic resistance patterns and availability of specific antimicrobial medicines when planning effective FN management pathways. ${ }^{13}$

\section{Diagnosis and Initial Investigation of FN}

Optimal management of FN requires consistent, evidencebased, definitions of the condition. No international consensus exists on the definitions of fever and neutropenia although common definitions of fever include $>38{ }^{\circ} \mathrm{C}$, $>38.3{ }^{\circ} \mathrm{C}$, or $>38.5{ }^{\circ} \mathrm{C}$, and for neutropenia are $<0.5$ $\mathrm{x} 10^{9} / \mathrm{L}$ or $<1.0 \times 10^{9} / \mathrm{L}$ and expected to fall to $<0.5 \times 10^{9} /$ L within $48 \mathrm{~h}^{14}$ Within the UK, the National Institute for Health and Care Excellence (NICE) guidelines advise the use of a neutrophil count of $\leq 0.5 \times 10^{9} / \mathrm{L}$ and either a fever $>38{ }^{\circ} \mathrm{C}$ or other signs or symptoms consistent with clinically significant sepsis. ${ }^{15}$ However, only $64 \%$ of UK centres used this definition in a 2017 audit. ${ }^{16}$ Furthermore, a recent trial conducted in Switzerland has suggested that a limit of $39{ }^{\circ} \mathrm{C}$ ear temperature is non-inferior to $38.5{ }^{\circ} \mathrm{C} .{ }^{17}$ Further work is needed to clarify the most appropriate definitions of $\mathrm{FN}$, and to facilitate consistency of use across the clinical and research community.

Blood cultures remain the gold standard test for diagnosis of blood stream infections in FN. While early studies found up to $22 \%$ of children with FN had a bacteraemia, more recent observational data indicate that bacteraemia rates may be lower than this and likely explained by the exclusion of common commensals unless cultured more than once. ${ }^{3}$ Not surprisingly, the diagnostic yield is highest when two or more blood cultures are taken prior to the first dose of antibiotics and, in the absence of a new fever or clinical instability, blood cultures beyond 48 hours of persistent fever have limited diagnostic utility. ${ }^{3}$ Data from a prospective, observational paediatric FN study found that almost $75 \%$ of blood cultures were positive within the first 24 hours of collection. Observational paediatric data also show as many as $17 \%$ of true blood 
stream infections in patients with a central venous catheter (CVC) are detected in cultures taken from a peripheral vein only, suggesting both CVC and peripheral vein cultures should be collected to optimise diagnosis. ${ }^{18}$ The quality of collection, including number of sets (aerobic and anaerobic) taken pre antibiotics and volume of blood have also been shown to impact the diagnostic yield of blood cultures in the general paediatric population, highlighting the importance of specific blood culture collection guidelines in $\mathrm{FN}{ }^{19}$

Rapid diagnostic technologies that expedite pathogen identification are, in theory, an important way to improve antibiotic use in FN. Despite the availability and use of these technologies for infection diagnosis in other areas of medicine, few studies have explored the clinical impact of these in the FN population. ${ }^{20}$ Concerns about polymerase chain reaction (PCR) based systems include that the panels used may not cover all of the organisms seen in this population, the sensitivity of the PCR means tiny amounts of bacterial nucleic acids or contamination create noisy results and not all antimicrobial resistance can be spotted in the circulating DNA. ${ }^{21}$

There is emerging evidence that PCR based tests for respiratory viruses can increase diagnostic accuracy in children with FN. In a study of nasopharyngeal samples obtained in 1044 episodes of FN in 525 children, multiplex PCR testing for 17 respiratory viruses revealed at least 1 respiratory virus in $46 \%$ of cases and respiratory virus as the sole pathogen(s) detected in $34 \%$ of episodes. ${ }^{22}$ The most common viruses detected were rhinovirus, respiratory syncytial virus, parainfluenza, influenza viruses, adenovirus and human metapneumovirus. The same researchers randomised 176 patients with $\mathrm{FN}$, negative bacterial cultures and favourable clinical evolution of their illness at $48 \mathrm{~h}$ between continuing antibiotics and stopping antibiotics in hospital, with no differences in duration of fever, days of hospitalisation and bacterial infections, no deaths and only one case of sepsis requiring intensive care admission in a patient continuing antibiotics. $^{23}$ With further data on safety and efficacy, such approaches may make an important contribution to AMR in the future.

Data on the role of PCR for detecting blood stream infections are more limited. In adult patients with FN, multiplex PCR systems reduced time to appropriate antibiotics but had limited impact on duration in two studies. ${ }^{24}$ In contrast, a randomised trial of BioFire Filmarray coupled with a comprehensive AMS program in adult patients, including $40 \%$ who were immunocompromised, did show reductions in both areas. ${ }^{25}$ While the data for rapid, molecular based diagnostics in paediatric FN are scant so far, these results highlight the importance of both diagnostic and AMS interventions to ensure appropriate use of these, often costly, tests.

Biomarkers to predict infection or severity of illness have been extensively explored in paediatric FN. ${ }^{1,26,27}$ However, while over 40 studies have investigated a range of biomarkers, most commonly procalcitonin, C-reactive protein and IL-6 and IL-8, very few clinical decision rules or risk stratification strategies incorporate these. The lack of validation and impact studies, combined with cost and availability, may in part explain this.

The role of diagnostic imaging, specifically fluorodeoxyglucose positron emission tomography (FDG-PET) combined with computed tomography (CT) or magnetic resonance imaging (MRI), for investigation of prolonged FN is also emerging as a potentially useful tool. ${ }^{28,29}$ A retrospective study of children with cancer and prolonged or recurrent FN found that, compared to conventional imaging, FDG-PET/CT identified additional sites of clinically significant infection/inflammation compared to conventional imaging. The study also showed that the FDG-PET results had a clinical impact in $80 \%$, leading to de-escalation or stopping of antibiotics in many patients. ${ }^{30}$ Routine use of FDG-PET has also been proposed as an adjunct to guiding treatment duration of invasive fungal infection in immunocompromised patients and has been shown to be cost-effective in this situation. ${ }^{31,32}$ The potential benefits of FDG-PET for prolonged or unexplained $\mathrm{FN}$ in children, in particular the identification of occult infection, needs to be balanced with availability and requirement for sedation in some patients.

\section{Principles/Concept of Risk Stratification in Paediatric FN}

Many groups have generated systems to stratify FN episodes at presentation, and during treatment, into low- or high-risk of infection-related adverse outcomes. ${ }^{33}$ The systems are intended to allow clinicians to alter the intensity, duration and consequently location of empiric therapy, in particular to select patients who are suitable for reduced intensity, often home-based, care. The systems tend to combine factors derived from the likely depth and duration of immunosuppression, episode-related elements of clinical presentation such as shock or hypoxia, 
and in the case of those choosing patients for out-ofhospital programmes, the patient's social situation. ${ }^{34,35}$ These are integrated to mean those who have received conditioning chemotherapy for a hematopoietic stem cell transplant, or live far from medical facilities without their own transport, or have arrived in hospital in septic shock would not be treated as out-patients. Although many riskstratification approaches have been proposed, few of them have proven effective in isolation. ${ }^{8}$ This may be overcome by embedding them into FN care pathways and taking a systems approach to implementation and evaluation. To be useful in practice, clinical decision rules should define at least $20 \%$ of patients as low risk; ${ }^{8}$ one study embedding clinical risk stratification into FN care pathways identified $27 \%$ of patients as "low risk" ${ }^{36}$ Other groups are conducting trials to determine if a biomarker-led approach may be even more effective than the clinically based stratification. ${ }^{37}$

\section{Optimising Antimicrobial Selection Empiric Antibiotics}

The choice of empirical antibacterial agents is derived from a knowledge of the expected incidence of particular bacteria, in part driven by risk stratification, the consequence of infection, and their likely antibacterial resistance. ${ }^{38}$ Gram positive organisms are identified in blood cultures more commonly than gram negative organisms ( $58 \%$ vs $42 \%$ ): the commonest pathogens identified are coagulase negative staphylococci (23\%), Enterobacteriaceae (23\%), viridans streptococci (13\%) and Pseudomonas aeruginosa (9\%). ${ }^{39}$ In most countries, there has been a historical evolution through intravenous dual-therapy (with an aminoglycoside) to stratified single-agent treatment with an intravenous antipseudomonal agent in those at higher risk of serious infection, and oral therapy with an antipseudomonal fluoroquinolone with or without a penicillin in those at lower risk of infection. This has been guided by many randomised clinical trials as agents have been introduced to the market, mostly in adult and all-age populations, and trials driven by the development of stratification systems which promote reduced intensity therapy in the low-risk groups. ${ }^{9,15,40}$

The "best guess" antibacterial therapies are then modified in the light of the developing clinical picture. Such changes have historically included planned progressive therapy; such as the addition of a glycopeptide if fever continues beyond 2-3 days. Clinical trials have demonstrated the lack of value of this, ${ }^{41-43}$ but the practice continues and highlights the need for robust AMS interventions such as pre-authorisations and implementation of clinical pathways encompassing the entire FN journey coupled with clinical audit and feedback. ${ }^{41-43}$ These interventions are critical as the increase in antimicrobial resistance (AMR) worldwide threatens the success of traditional empiric FN antibiotic choices. An international study across 15 paediatric cancer centres in eight countries found high rates of piperacillin-tazobactam resistance among some important Gram-negative pathogens including, Escherichia coli, Klebsiella pneumoniae and P. aeruginosa. ${ }^{44}$ As the incidence of AMR will vary between hospitals and even individual departments, empiric FN choices should be informed by local antibiograms in consultation with microbiology and infectious diseases specialists. In studies of children with cancer, independent risk factors for AMR include prior antibiotic exposure and hospitalisation and AMR infections are associated with adverse outcomes including ICU admission, prolonged hospitalisation and death. ${ }^{45,46}$ For patients travelling from overseas for treatment, the incidence of AMR at the sending centre may be unknown and the potential for AMR may be a particular consideration. To combat these concerns, diagnostic and AMS principles should be embedded within FN guidelines to ensure the right investigations are done and interpretation of these inform the right antibiotic choice and duration. ${ }^{47,48}$ Comprehensive and collaborative AMS interventions have been shown to reduce antibiotic exposure in immunocompromised patients without compromising patient safety and are vital to limiting the impact of AMR. ${ }^{47,49-51}$

\section{Timing of Antibiotics}

As well as choice of antibiotics, the time to administration (TTA) of empirical antibiotics is expected to influence the outcome for patients with bacteraemia or sepsis. Within existing research, TTA is most commonly defined as time from arrival in hospital to administration of antibiotics although in some studies is defined as onset of fever to antibiotic administration. ${ }^{52}$ Adult FN guidelines in Europe and America advocate a TTA of $<1$ hour; most paediatric guidelines are not specific on this point but a time of $<1$ hour is generally considered as good practice among treating physicians. ${ }^{53,54}$ A systematic review of TTA in adult and paediatric FN episodes was suggestive of an increased risk of death, intensive care admission and sepsis with a longer TTA but triage bias (in which patients 
who are more unwell receive faster treatment) was noted. $^{52}$ Despite this lack of precise evidence, TTA is considered a measure of quality of care in paediatric FN and a number of different, successful approaches to reduce TTA have been described. ${ }^{55}$ These include staff training interventions, guidelines, checklists and treatment algorithms. $^{56}$

\section{Viral and Fungal Infections}

As in many instances of paediatric fever, viral infections are common within the paediatric oncology population, although, due to underlying immunosuppression, may present atypically. ${ }^{57}$ Obtaining a history of contact with infectious individuals and consideration of risk factors for viral reactivation (ie allogeneic HSCT) is important and will guide diagnostics, preferably molecular based. Empirical anti-viral therapy, eg oseltamivir, may be considered in children with an influenza-like illness, during periods of high population prevalence. Where viral infections are suspected, local or international guidelines for management should be followed. ${ }^{58-63}$

The paediatric oncology patients who are most at risk of invasive fungal infections (IFI) are those with severe and prolonged neutropenia, often those with acute leukaemias or receiving HSCT. Additional factors associated with IFI include high-dose steroid exposure, acute and chronic GvHD and increasing age. ${ }^{64}$ Particular attention should be paid to the clinical history, in particular symptoms such as haemoptysis, chest pain, sinus pain, dental pain, or skin lesions. Diagnostic markers such as aspergillus antigen, candida antigen, and beta-D-glucan have variable performances and tissue or fluid culture or histopathology remains the gold standard. ${ }^{9}$ Imaging with CT chest \pm sinuses, ultrasound of the abdomen to exclude hepatosplenic lesions and fundoscopy are recommended. Empirical treatment with anti-fungal agents is usually considered in patients at higher risk of IFI between days 3 and 5 when there has been inadequate response to initial antibiotic therapies. However, this may be indicated early in very high-risk individuals (eg post-allogeneic HSCT, induction therapy for acute leukaemia) or in those with symptoms or signs of invasive fungal disease. A review of international antifungal treatment guidelines has shown that these are varied in quality and recommendations; thus, no single national or international guideline can be recommended. ${ }^{65}$ Preemptive antifungal therapy in the face of evidence of IFI can be considered as a strategy in place of empirical antifungal therapy for children with $\mathrm{FN}$ at risk of IFI but this approach is currently not widely practised and is dependent on rapid access to pulmonary CT imaging, galactomannan test results and, ideally, the ability to undertake bronchoscopies with bronchoalveolar lavage. $^{66}$

\section{Impact of Prophylaxis}

Antimicrobial selection for FN should also take into consideration concurrent prophylaxis. A systematic review of RCTs, including 13 paediatric studies, found fluoroquinolone prophylaxis with levofloxacin reduced episodes of bacteraemia, fever and FN. ${ }^{67}$ However there was no difference in overall mortality and not surprisingly, an increase in fluoroquinolone resistance. Prophylaxis guidelines for prevention of viral, Pneumocystis jirovecii pneumonia, and invasive fungal infections are available and beyond the scope of this review. ${ }^{58,65,68-71}$

\section{Duration of Treatment}

The duration of empirical antibiotic therapy remains contentious. An early trial, looking at discontinuation after a negative blood culture result compared with continuing to count recovery, showed an increased risk of death in the early stopping arm. ${ }^{72}$ This understandably unsettled the oncology world, and led to a standard being set of continuing therapy until the patient was afebrile, free of documented infection, and had a neutrophil count above a specified threshold (often $0.5 \times 10^{9} / \mathrm{L}$ ).

While there are robust paediatric data for reduced intensity therapy such as oral antibiotics or home-based treatments for children with low-risk FN, the specific evidence for antibiotic duration in either low- or highrisk groups is scant. ${ }^{73}$ For children with unexplained fever, there are generally two approaches to duration namely (i) continue until clear signs of marrow recovery or (ii) continue until patient is stable and afebrile, irrespective of neutrophil count or expected duration of neutropenia. ${ }^{15,74,75}$ Despite the frequency with which FN occurs in both adult and paediatric cancer patients, as few as eight RCTs have specifically compared these two approaches, with only one focusing on high-risk patients. $^{76}$ While children are well represented in these studies, there is marked heterogeneity in underlying risk status, type of malignancy, definition of clinical failure and time of randomisation of included participants and most studies were conducted before the year 2000. Acknowledging these limitations, a Cochrane review found no significant differences in rates of mortality or 
clinical failures between short or long-course empiric antibiotic therapy arms and fewer antibiotic days (by 3-7 days) in the former. ${ }^{76}$ Well conducted, prospective trials that address antibiotic duration in paediatric patients with high-risk FN and that challenge the dogma of continuing antibiotics until count recovery are urgently required to inform guidelines and clinical practice internationally.

The lack of solid data on when to stop empiric treatment mirrors the lack of data supporting the duration of focused treatment in identified infections, and the lack of attention to this topic in paediatrics generally. Traditional approaches have tended to the decimal or the lunar - with treatments being a multiple of 7 days, or occasionally 10 . For central line associated blood stream infections (CLABSIs), the duration of therapy may depend on the organism identified and whether or not the line remains in situ, guided by local policies. More individualised approaches, where the duration of antimicrobials is guided by inflammatory or infectious markers, are currently under investigation in large trials of immunocompetent children, such as the Batch study in the UK. ${ }^{77}$

When considering the duration of therapy in $\mathrm{FN}$, both the duration of antimicrobials and the duration of hospitalisation should be considered. Over the years, various regimes have been evaluated, including multiple combinations of locations (hospital vs home) and route of antibiotic administration (IV vs oral). A 2016 systematic review of these approaches in paediatric patients with low-risk FN found that reduced intensity therapies were safe with low rates of treatment failure. ${ }^{73}$ An implementation study from Australia similarly showed a significant reduction in hospital length (from 4.0 to 1.5 days) with low readmission rates (13\%) and no adverse outcomes in patients managed on a formal lowrisk FN program. ${ }^{78}$ This program is being scaled nationwide and has been adapted for use in the UK. Beyond safety, home-based FN care has been shown to improve quality of life, and reduce healthcare costs, which have been estimated at between US $\$ 5600$ and $\$ 11,700$ per episode of FN, depending on the regimes used for comparison and the country in which the research was performed. ${ }^{79-81}$

\section{Current Best Practice and Future Directions in FN}

Whilst the above evidence review shows that there are still acceptable variations in practice owing to a number of unanswered questions about FN management, there is broad agreement on the key considerations in FN care. Local centres should have policies and care pathways for FN management that cover the features included in Box 1 and Figure 1. Box 2 shows areas for further research in paediatric FN.

Implementing the findings of research in this area has met challenges in terms of effecting change in healthcare provision. Within the UK, repeated national audits of paediatric FN management have found slow changes in practice, despite clear national guidelines. Some of these issues may relate to previous healthcare professional experiences and approaches to risk stratification. ${ }^{82}$ However, our experiences of the recent COVID-19 pandemic have highlighted the ability to effect rapid implementation of new practices within FN management, based on previous research. ${ }^{83}$ Thus, future research may focus also on the key aspects of implementation science in this area.

\section{Conclusion}

Although the importance of recognition and careful management of FN has long been known, modern best practice demands prompt recognition, early treatment according to risk stratified pathways and attention to the choice of empiric antibiotics, role of oral as well as intravenous

Box I Key Features of FN Policies and Guidelines

- Definitions of FN

- Early recognition of FN

- Routine investigations for suspected FN including peripheral blood cultures even if central venous access device present

- Rapid administration of broad-spectrum antibiotics

- Recommended empirical antibiotic regimes

- Risk stratification with defined management pathways for

- Low-risk episodes of care

- High-risk episodes of care

- Guidelines on treatment modification including investigation and initiation of antifungal therapy

- Guidelines on duration of treatment by risk group 


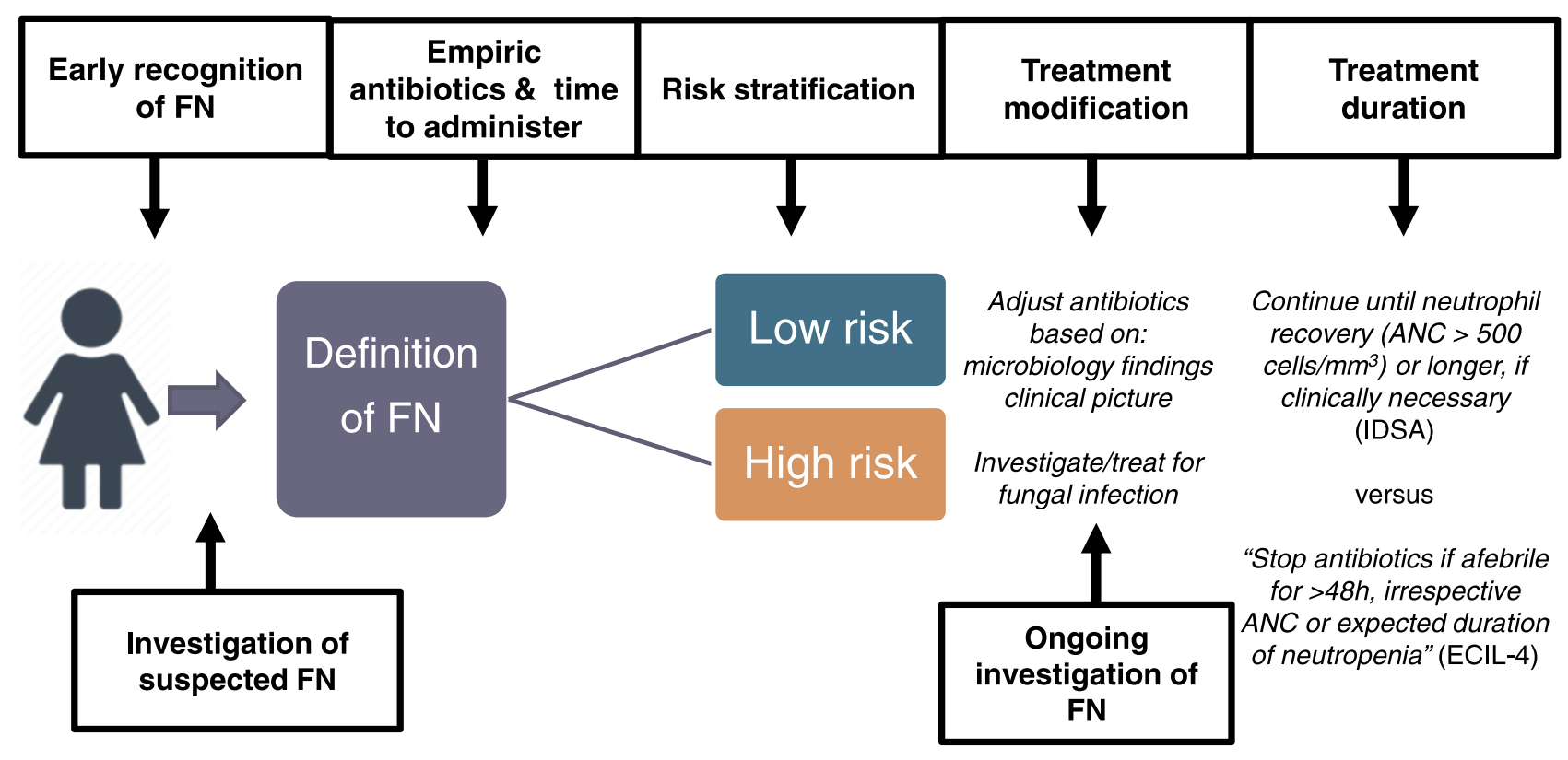

Figure I Paediatric FN patient pathway and opportunities for intervention and optimisation. ${ }^{74,75}$

antibiotics, place of care and duration of treatment in order to give optimal treatment to high-risk patients whilst reducing hospital stay where possible and exercising good
AMS for all patients. Future research will be important to close current gaps in knowledge to further refine current treatment protocols and optimise ways of effecting

Box 2 Research Gaps in Paediatric FN 910

\begin{tabular}{l} 
- Optimal definition of fever and neutropenia \\
- Routine investigations for suspected FN \\
- Incremental value of a peripheral blood culture in addition to CVC cultures of adequate volume in children with FN \\
- Utility of new serum biomarkers in children with FN \\
- Impact of novel biomarkers or point of care tests on antimicrobial selection and duration, including role of PCR for \\
respiratory viruses \\
- Rapid administration of broad-spectrum antibiotics, including optimal TTA \\
- Which patients with FN will benefit from antibiotic administration within I hr \\
- Recommended empirical antibiotic regimes \\
- Optimal empirical antibiotics in low-risk FN \\
- Risk stratification and care pathways \\
- Developing a validated high-risk stratification schema for paediatric FN \\
- Implementation and impact (clinical, economic and QoL) of risk stratification pathways \\
- Optimal type and frequency of re-evaluation for paediatric outpatients with low-risk FN \\
- Treatment modification \\
- Optimal frequency of blood culture sampling in persistently febrile paediatric patients with neutropenia who are either \\
clinically stable or unstable \\
- Optimal investigation and treatment for viral and fungal infections in children with FN \\
- Safety and efficacy of short course antibiotics in children with high-risk FN \\
- Safety and efficacy of targeted therapy for documented clinical infection \\
- Should diagnostic and therapeutic approaches differ for prolonged continuous fever vs recurrent fever during FN \\
Optimal duration of antibiotic therapy \\
- Optimal treatment duration for microbiologically documented sterile site infections during FN \\
- Guidelines on duration of treatment by risk group \\
- Cost-effectiveness of different approaches to managing paediatric FN \\
\hline
\end{tabular}


adoption of such improvements. It remains to be seen whether rapid diagnostic PCR-based techniques will be able to revolutionise pathogen detection, antibiotic selection and antimicrobial stewardship.

\section{Author Contributions}

All authors made a significant contribution to the work reported, whether that is in the conception, study design, execution, acquisition of data, analysis and interpretation, or in all these areas; took part in drafting, revising or critically reviewing the article; gave final approval of the version to be published; have agreed on the journal to which the article has been submitted; and agree to be accountable for all aspects of the work.

\section{Funding}

The authors have received no funding for the writing of this review.

\section{Disclosure}

JCC is supported by the Royal Marsden Cancer Charity and by National Health Service funding to the National Institute for Health Research Biomedical Research Centre of The Royal Marsden Hospital. JCC also served on an advisory board and reports educational presentation fees from Bayer. GMH reports grants from Medical Research Future Fund and Victorian Cancer Agency, outside the submitted work. JEM is supported by an NIHR Clinical Lectureship. The authors report no other conflicts of interest in this work.

\section{References}

1. Arif T, Phillips RS. Updated systematic review and meta-analysis of the predictive value of serum biomarkers in the assessment and management of fever during neutropenia in children with cancer. Pediatr Blood Cancer. 2019;66(10):e27887. doi:10.1002/pbc.27887

2. Alexander S, Fisher BT, Gaur AH, et al. Effect of levofloxacin prophylaxis on bacteremia in children with acute leukemia or undergoing hematopoietic stem cell transplantation: a randomized clinical trial. JAMA. 2018;320(10):995-1004. doi:10.1001/jama.2018.12512

3. Haeusler GM, De Abreu Lourenco R, Clark H, et al. Diagnostic yield of initial and consecutive blood cultures in children with cancer and febrile neutropenia. J Pediatric Infect Dis Soc. 2020. doi:10.1093/ jpids/piaa029

4. Castagnola E, Fontana V, Caviglia I, et al. A prospective study on the epidemiology of febrile episodes during chemotherapy-induced neutropenia in children with cancer or after hemopoietic stem cell transplantation. Clin Infect Dis. 2007;45(10):1296-1304. doi:10.1086/522533

5. Herd F, Bate J, Chisholm J, Johnson E, Phillips B. Variation in practice remains in the UK management of paediatric febrile neutropenia. Arch Dis Child. 2016;101(4):410-411. doi:10.1136/archdischild-2015-310294

6. Haeusler GM, Slavin MA, Bryant PA, Babl FE, Mechinaud F, Thursky KA. Management of fever and neutropenia in children with cancer: a survey of Australian and New Zealand practice. J Paediatr Child Health. 2018;54(7):761-769. doi:10.1111/jpc.13899
7. Duncan C, Chisholm JC, Freeman S, Riley U, Sharland M, PritchardJones K. A prospective study of admissions for febrile neutropenia in secondary paediatric units in South East England. Pediatr Blood Cancer. 2007;49(5):678-681. doi:10.1002/pbc.21041

8. Haeusler GM, Thursky KA, Slavin MA, et al. Risk stratification in children with cancer and febrile neutropenia: a national, prospective, multicentre validation of nine clinical decision rules. EClinicalMedicine. 2020;18:100220. doi:10.1016/j.eclinm.2019.11.013

9. Lehrnbecher T, Robinson P, Fisher B, et al. Guideline for the management of fever and neutropenia in children with cancer and hematopoietic stem-cell transplantation recipients: 2017 update. JCO. 2017;35(18):2082-2094. doi:10.1200/JCO.2016.71.7017

10. Lehrnbecher T, Phillips R, Alexander S, et al. Guideline for the management of fever and neutropenia in children with cancer and/ or undergoing hematopoietic stem-cell transplantation. J Clin Oncol. 2012;30(35):4427-4438. doi:10.1200/JCO.2012.42.7161

11. Allaway Z, Phillips RS, Thursky KA, Haeusler GM. Nonneutropenic fever in children with cancer: a scoping review of management and outcome. Pediatr Blood Cancer. 2019;66(6):e27634. doi:10.1002/pbc. 27634

12. Morgan JE. Fifteen minute consultation: fever in children being treated for cancer. Arch Dis Child. 2019;104(3):124-128. doi:10.11 36/archdischild-2017-314718

13. Israels $T$, Renner $L$, Hendricks $M$, Hesseling $P$, Howard $S$, Molyneux E. SIOP PODC: recommendations for supportive care of children with cancer in a low-income setting. Pediatr Blood Cancer. 2013;60(6):899-904. doi:10.1002/pbc.24501

14. Haeusler GM, Phillips RS, Lehrnbecher T, Thursky KA, Sung L, Ammann RA. Core outcomes and definitions for pediatric fever and neutropenia research: a consensus statement from an international panel. Pediatr Blood Cancer. 2015;62(3):483-489. doi:10.1002/pbc.25335

15. NICE. CG151 neutropenic sepsis: full guideline. NICE. Available from: http://www.nice.org.uk/. Accessed September 25, 2013.

16. Morgan JE, Phillips B. Winter 2017 children's cancer and leukaemia group febrile neutropenia audit. Arch Dis Child. 2018; archdischild2018-315249. doi:10.1136/archdischild-2018-315249

17. Koenig C, Bodmer N, Agyeman PKA, et al. $39 \cdot 0^{\circ} \mathrm{C}$ versus $38 \cdot 5^{\circ} \mathrm{C}$ ear temperature as fever limit in children with neutropenia undergoing chemotherapy for cancer: a multicentre, cluster-randomised, multiple-crossover, non-inferiority trial. Lancet Child Adolesc Health. 2020;4(7):495-502. doi:10.1016/S2352-4642(20)30092-4

18. Rodríguez L, Ethier M-C, Phillips B, Lehrnbecher T, Doyle J, Sung L. Utility of peripheral blood cultures in patients with cancer and suspected blood stream infections: a systematic review. Support Care Cancer. 2012;20(12):3261-3267. doi:10.1007/s00520-012-1471-2

19. Feghaly REE, Chatterjee J, Dowdy K, et al. A quality improvement initiative: reducing blood culture contamination in a children's hospital. Pediatrics. 2018;142(4):e20180244. doi:10.1542/peds.2018-0244

20. Stevenson M, Pandor A, James MS, et al. Sepsis: the LightCycler SeptiFast test MGRADE ${ }^{\circledR}$, SepsiTest and IRIDICA BAC BSI assay for rapidly identifying bloodstream bacteria and fungi - a systematic review and economic evaluation. Health Technol Assess (Rockv). 2016;20(46). doi:10.3310/hta20460

21. Guido M, Quattrocchi M, Zizza A. Molecular approaches in the diagnosis of sepsis in neutropenic patients with haematological malignances. J Prev Med Hyg. 2012;53(2):104-108.

22. Torres JP, De la Maza V, Kors L, et al. Respiratory viral infections and coinfections in children with cancer, fever and neutropenia: clinical outcome of infections caused by different respiratory viruses. Pediatr Infect Dis J. 2016;35(9):949-954. doi:10.1097/ INF.0000000000001209

23. Santolaya ME, Alvarez AM, Acuña M, et al. Efficacy and safety of withholding antimicrobial treatment in children with cancer, fever and neutropenia, with a demonstrated viral respiratory infection: a randomized clinical trial. Clin Microbiol Infect. 2017;23(3): 173-178. doi:10.1016/j.cmi.2016.11.001 
24. Rosa R, Suarez JF, Bravo G, et al. Challenges in antimicrobial stewardship: rapid diagnostics and optimization of therapy among immunocompromised patients. Open Forum Infect Dis. 2019;6(7). doi:10.1093/ofid/ofz239

25. Buss BA, Baures TJ, Yoo M, et al. Impact of a multiplex PCR assay for bloodstream infections with and without antimicrobial stewardship intervention at a cancer hospital. Open Forum Infect Dis. 2018;5 (10). doi:10.1093/ofid/ofy258

26. Phillips RS, Wade R, Lehrnbecher T, Stewart LA, Sutton AJ. Systematic review and meta-analysis of the value of initial biomarkers in predicting adverse outcome in febrile neutropenic episodes in children and young people with cancer. BMC Med. 2012;10(1):6 doi:10.1186/1741-7015-10-6

27. Haeusler GM, Carlesse F, Phillips RS. An updated systematic review and meta-analysis of the predictive value of serum biomarkers in the assessment of fever during neutropenia in children with cancer. Pediatr Infect Dis J. 2013;32(10):e390-6. doi:10.1097/INF.0b013e 31829ae38d

28. Douglas AP, Thursky KA, Worth LJ, Harrison SJ, Hicks RJ, Slavin MA. FDG-PET/CT in managing infection in patients with hematological malignancy: clinician knowledge and experience in Australia. Leuk Lymphoma. 2019;60(10):2471-2476. doi:10.1080/ 10428194.2019.1590571

29. Douglas A, Lau E, Thursky K, Slavin M. What, where and why: exploring fluorodeoxyglucose-PET's ability to localise and differentiate infection from cancer. Curr Opin Infect Dis. 2017;30(6):552-564. doi:10.1097/QCO.0000000000000405

30. Wang SS, Mechinaud F, Thursky K, Cain T, Lau E, Haeusler GM. The clinical utility of fluorodeoxyglucose-positron emission tomography for investigation of fever in immunocompromised children. J Paediatr Child Health. 2018;54(5):487-492. doi:10.1111/jpc.13809

31. Fernández-Cruz A, Lewis RE, Kontoyiannis DP. How long do we need to treat an invasive mold disease in hematology patients? factors influencing duration of therapy and future questions. Clin Infect Dis. 2020;71(3):685-692. doi:10.1093/cid/ciz1195

32. Koh KC, Slavin MA, Thursky KA, et al. Impact of fluorine-18 fluorodeoxyglucose positron emission tomography on diagnosis and antimicrobial utilization in patients with high-risk febrile neutropenia. Leuk Lymphoma. 2012;53(10):1889-1895. doi:10.3109/10428194.20 12.677533

33. Haeusler GM, Thursky KA, Slavin MA, et al. External validation of six pediatric fever and neutropenia clinical decision rules. Pediatr Infect Dis J. 2018;37(4):329-335. doi:10.1097/INF.0000000000001777

34. Haeusler GM, Phillips R, Slavin MA, et al. Re-evaluating and recalibrating predictors of bacterial infection in children with cancer and febrile neutropenia. EClinicalMedicine. 2020;23:100394. doi:10.10 16/j.eclinm.2020.100394

35. Phillips B, Morgan JE, Haeusler GM, Riley RD, PICNICC Collaborative. Individual participant data validation of the PICNICC prediction model for febrile neutropenia. Arch Dis Child. 2020;105(5):439-445. doi:10.1136/archdischild-2019-317308

36. Dommett R, Geary J, Freeman S, et al. Successful introduction and audit of a step-down oral antibiotic strategy for low risk paediatric febrile neutropaenia in a UK, multicentre, shared care setting. Eur J Cancer. 2009;45(16):2843-2849. doi:10.1016/j.ejca.2009.06.003

37. ISRCTN. ISRCTN13185152: Is It Practical to Perform a Study to Manage Antibiotic Delivery in Febrile Neutropenia for Children and Young People Undergoing Treatment with Anti-Cancer Drugs with the Routine Serial Use of PCT Measurements? 2020. doi:10.1186/ISRCTN13185152

38. Haeusler GM, Sung L, Ammann RA, Phillips B. Management of fever and neutropenia in paediatric cancer patients: room for improvement? Curr Opin Infect Dis. 2015;28(6):532-538. doi:10. 1097/QCO.0000000000000208

39. Mikulska M, Viscoli C, Orasch C, et al. Aetiology and resistance in bacteraemias among adult and paediatric haematology and cancer patients. J Infect. 2014;68(4):321-331. doi:10.1016/j.jinf.2013.12.006
40. Vidal L, Dor IB, Paul M, et al. Oral versus intravenous antibiotic treatment for febrile neutropenia in cancer patients. Cochrane Database Syst Rev. 2013;(10). doi:10.1002/14651858.CD003992. pub3

41. Cometta A, Kern WV, De Bock R, et al. Vancomycin versus placebo for treating persistent fever in patients with neutropenic cancer receiving piperacillin-tazobactam monotherapy. Clin Infect Dis 2003;37(3):382-389. doi:10.1086/376637

42. Erjavec Z, de Vries-hospers HG, Laseur M, Halie RM, Daenen S. A prospective, randomized, double-blinded, placebo-controlled trial of empirical teicoplanin in febrile neutropenia with persistent fever after imipenem monotherapy. J Antimicrob Chemother. 2000;45 (6):843-849. doi:10.1093/jac/45.6.843

43. Pizzo PA, Robichaud KJ, Gill FA, Witebsky FG. Empiric antibiotic and antifungal therapy for cancer patients with prolonged fever and granulocytopenia. Am J Med. 1982;72(1):101-111. doi:10.1016/ 0002-9343(82)90594-0

44. Mesini A, Carlesse F, Ammann R, et al. Proportions of antimicrobial resistant BSIs in children receiving antineoplastic chemotherapy (AC) or hemopoietic stem cell transplant (HSCT): preliminary results from an international multicenter study. In: 29th ECCMID; 2019. Abstract No 2054.

45. Levene I, Castagnola E, Haeusler GM. Antibiotic-resistant gram-negative blood stream infections in children with cancer: a review of epidemiology, risk factors, and outcome. Pediatr Infect Dis J. 2018;37(5):495-498. doi:10.1097/INF.000000000000 1938

46. Haeusler GM, Levene I. Question 2: what are the risk factors for antibiotic resistant gram-negative bacteraemia in children with cancer? Arch Dis Child. 2015;100(9):895-898. doi:10.1136/archdischild-2015-309175

47. Pillinger KE, Bouchard J, Withers ST, et al. Inpatient antibiotic stewardship interventions in the adult oncology and hematopoietic stem cell transplant population: a review of the literature. Ann Pharmacother. 2020;54(6):594-610. doi:10.1177/1060028019890 886

48. Messacar K, Parker SK, Todd JK, Dominguez SR, Kraft CS. Implementation of rapid molecular infectious disease diagnostics: the role of diagnostic and antimicrobial stewardship. $J$ Clin Microbiol. 2017;55(3):715-723. doi:10.1128/JCM.02264-16

49. Hennig S, Staatz CE, Natanek D, et al. Antimicrobial stewardship in paediatric oncology: impact on optimising gentamicin use in febrile neutropenia. Pediatr Blood Cancer. 2018;65(2):e26810. doi:10.1002/ pbc. 26810

50. Santiago-García B, Rincón-López EM, Ponce Salas B, et al. Effect of an intervention to improve the prescription of antifungals in pediatric hematology-oncology. Pediatr Blood Cancer. 2020;67(4):e27963. doi:10.1002/pbc. 27963

51. Karandikar MV, Milliren CE, Zaboulian R, et al. Limiting vancomycin exposure in pediatric oncology patients with febrile neutropenia may be associated with decreased vancomycin-resistant enterococcus incidence. J Pediatric Infect Dis Soc. 2020;9(4):428-436. doi:10.10 93/jpids/piz064

52. Koenig C, Schneider C, Morgan JE, Ammann RA, Sung L, Phillips B. Association of time to antibiotics and clinical outcomes in patients with fever and neutropenia during chemotherapy for cancer: a systematic review. Support Care Cancer. 2020;28 (3):1369-1383. doi:10.1007/s00520-019-04961-4

53. Klastersky J, de Naurois J, Rolston K, et al. Management of febrile neutropaenia: ESMO clinical practice guidelines. Ann Oncol. 2016;27(suppl 5):v111-v118. doi:10.1093/annonc/mdw325

54. Taplitz RA, Kennedy EB, Bow EJ, et al. Outpatient management of fever and neutropenia in adults treated for malignancy: American society of clinical oncology and infectious diseases society of america clinical practice guideline update. J Clin Oncol. 2018;36 (14):1443-1453. doi:10.1200/JCO.2017.77.6211 
55. McCavit TL, Winick N. Time-to-antibiotic administration as a quality of care measure in children with febrile neutropenia: a survey of pediatric oncology centers. Pediatr Blood Cancer. 2012;58(2):3 03-305. doi: $10.1002 /$ pbc. 23148

56. Koenig C, Schneider C, Morgan JE, Ammann RA, Sung L, Phillips B. Interventions aiming to reduce time to antibiotics (TTA) in patients with fever and neutropenia during chemotherapy for cancer (FN), a systematic review. Support Care Cancer. 2020;28 (5):2369-2380. doi:10.1007/s00520-019-05056-w

57. Agyeman P, Kontny U, Nadal D, et al. A prospective multicenter study of microbiologically defined infections in pediatric cancer patients with fever and neutropenia: Swiss pediatric oncology group 2003 fever and neutropenia study. Pediatr Infect Dis J. 2014;33(9): e219-225. doi:10.1097/INF.0000000000000326

58. Styczynski J, Reusser P, Einsele H, et al. Management of HSV, VZV and EBV infections in patients with hematological malignancies and after SCT: guidelines from the second European conference on infections in leukemia. Bone Marrow Transplant. 2009;43(10):757-770. doi:10.1038/bmt.2008.386

59. Ward KN, Hill JA, Hubacek P, et al. Guidelines from the 2017 European conference on infections in leukaemia for management of HHV-6 infection in patients with hematologic malignancies and after hematopoietic stem cell transplantation. Haematologica. 2019;104 (11):2155-2163. doi:10.3324/haematol.2019.223073

60. Ljungman P, de la Camara R, Robin C, et al. Guidelines for the management of cytomegalovirus infection in patients with haematological malignancies and after stem cell transplantation from the 2017 European conference on infections in leukaemia (ECIL 7). Lancet Infect Dis. 2019;19(8):e260-e272. doi:10.1016/S1473-3099(19) 30107-0

61. Matthes-Martin S, Feuchtinger T, Shaw PJ, et al. European guidelines for diagnosis and treatment of adenovirus infection in leukemia and stem cell transplantation: summary of ECIL-4 (2011). Transpl Infect Dis. 2012;14(6):555-563. doi:10.1111/tid.12022

62. Hirsch HH, Martino R, Ward KN, Boeckh M, Einsele H, Ljungman P. Fourth European conference on infections in leukaemia (ECIL-4): guidelines for diagnosis and treatment of human respiratory syncytial virus, parainfluenza virus, metapneumovirus, rhinovirus, and coronavirus. Clin Infect Dis. 2013;56(2):258-266. doi:10.1093/cid/cis844

63. Engelhard D, Mohty B, de la Camara R, Cordonnier C, Ljungman P. European guidelines for prevention and management of influenza in hematopoietic stem cell transplantation and leukemia patients: summary of ECIL-4 (2011), on behalf of ECIL, a joint venture of EBMT, EORTC, ICHS, and ELN. Transpl Infect Dis. 2013;15(3):219-232. doi:10.1111/tid.12054

64. Fisher BT, Robinson PD, Lehrnbecher T, et al. Risk factors for invasive fungal disease in pediatric cancer and hematopoietic stem cell transplantation: a systematic review. J Pediatric Infect Dis Soc. 2018;7(3):191-198. doi:10.1093/jpids/pix030

65. Morgan JE, Hassan H, Cockle JV, Lethaby C, James B, Phillips RS. Critical review of current clinical practice guidelines for antifungal therapy in paediatric haematology and oncology. Support Care Cancer. 2016. doi:10.1007/s00520-016-3412-y

66. Groll AH, Castagnola E, Cesaro S, et al. Fourth European conference on infections in leukaemia (ECIL-4): guidelines for diagnosis, prevention, and treatment of invasive fungal diseases in paediatric patients with cancer or allogeneic haemopoietic stem-cell transplantation. Lancet Oncol. 2014;15(8):e327-340. doi:10.1016/ S1470-2045(14)70017-8

67. Egan G, Robinson PD, Martinez JPD, et al. Efficacy of antibiotic prophylaxis in patients with cancer and hematopoietic stem cell transplantation recipients: a systematic review of randomized trials. Cancer Med. 2019;8(10):4536-4546. doi:10.1002/cam4.2395
68. Lehrnbecher T, Fisher BT, Phillips B, et al. Clinical practice guideline for systemic antifungal prophylaxis in pediatric patients with cancer and hematopoietic stem-cell transplantation recipients. JCO. 2020;38(27):3205-3216. doi:10.1200/JCO.20.00158

69. Bate J, Baker S, Breuer J, et al. PEPtalk2: results of a pilot randomised controlled trial to compare VZIG and aciclovir as postexposure prophylaxis (PEP) against chickenpox in children with cancer. Arch Dis Child. 2019;104(1):25-29. doi:10.1136/archdischild-2017-314212

70. Proudfoot R, Cox R, Phillips B, Wilne S. Guidelines for the prophylaxis of pneumocystis jirovecii pneumonia (PJP) in children with solid tumours. Child Cancer Leukaemia Group. 2016;83.

71. Stern A, Green H, Paul M, Vidal L, Leibovici L. Prophylaxis for pneumocystis pneumonia (PCP) in non-HIV immunocompromised patients. Cochrane Database Syst Rev. 2014;2014(10). doi:10.1002/ 14651858.CD005590.pub3

72. Pizzo PA, Robichaud KJ, Gill FA, et al. Duration of empiric antibiotic therapy in granulocytopenic patients with cancer; 1979. Available from: http://onlinelibrary.wiley.com/o/cochrane/clcentral/articles/783/ CN-00020783/frame.html. Accessed September 25, 2013.

73. Morgan JE, Cleminson J, Atkin K, Stewart LA, Phillips RS. Systematic review of reduced therapy regimens for children with low risk febrile neutropenia. Support Care Cancer. 2016;24(6): 2651-2660. doi:10.1007/s00520-016-3074-9

74. Freifeld AG, Bow EJ, Sepkowitz KA, et al. Clinical practice guideline for the use of antimicrobial agents in neutropenic patients with cancer: 2010 update by the infectious diseases society of America. Clin Infect Dis. 2011;52(4):427-431. doi:10.1093/cid/ciq147

75. Averbuch D, Orasch C, Cordonnier C, et al. European guidelines for empirical antibacterial therapy for febrile neutropenic patients in the era of growing resistance: summary of the 2011 4th European conference on infections in leukemia. Haematologica. 2013;98 (12):1826-1835. doi:10.3324/haematol.2013.091025

76. Stern A, Carrara E, Bitterman R, Yahav D, Leibovici L, Paul M. Early discontinuation of antibiotics for febrile neutropenia versus continuation until neutropenia resolution in people with cancer. Cochrane Database Syst Rev. 2019;1:CD012184. doi:10.1002/ 14651858.CD012184.pub2

77. ISRCTN. ISRCTN11369832: Using Procalcitonin to Guide Duration of Antibiotics. 2017. doi:10.1186/ISRCTN11369832

78. Haeusler GM, Gaynor L, Teh B, et al. Home-based care of low-risk febrile neutropenia in children-an implementation study in a tertiary paediatric hospital. Support Care Cancer. 2020;29(3):1609-1617. doi:10.1007/s00520-020-05654-z

79. Mustafa MM, Aquino VM, Pappo A, Tkaczewski I, Buchanan GR. A pilot study of outpatient management of febrile neutropenic children with cancer at low risk of bacteremia. J Pediatr. 1996;128 (6):847-849. doi:10.1016/S0022-3476(96)70339-6

80. Teuffel O, Amir E, Alibhai SMH, Beyene J, Sung L. Costeffectiveness of outpatient management for febrile neutropenia in children with cancer. Pediatrics. 2011;127(2):e279-286. doi:10. 1542/peds.2010-0734

81. Orme L, Babl F, Barnes C, Barnett P, Donath S, Ashley D. Outpatient versus inpatient IV antibiotic management for pediatric oncology patients with low risk febrile neutropenia: a randomised trial. Pediatr Blood Cancer. 2014;61(8):1427-1433. doi:10.1002/pbc.25 012

82. Morgan JE, Phillips B, Stewart LA, Atkin K. Quest for certainty regarding early discharge in paediatric low-risk febrile neutropenia: a multicentre qualitative focus group discussion study involving patients, parents and healthcare professionals in the UK. BMJ Open. 2018;8(5):e020324. doi:10.1136/bmjopen-2017-020324

83. Phillips B, Morgan JE. Meta-analytic validation of new 'AUS' febrile neutropenia risk score. Pediatr Blood Cancer. 2021;e28580. doi:10.1002/pbc. 28580 


\section{Publish your work in this journal}

Infection and Drug Resistance is an international, peer-reviewed openaccess journal that focuses on the optimal treatment of infection (bacterial, fungal and viral) and the development and institution of preventive strategies to minimize the development and spread of resistance. The journal is specifically concerned with the epidemiology of

Submit your manuscript here: https://www.dovepress.com/infection-and-drug-resistance-journa| antibiotic resistance and the mechanisms of resistance development and diffusion in both hospitals and the community. The manuscript management system is completely online and includes a very quick and fair peerreview system, which is all easy to use. Visit http://www.dovepress.com/ testimonials.php to read real quotes from published authors. 